\title{
Preliminary analysis of the benefits of different irradiation types on patients with postoperative locoregional recurrence of esophageal cell squamous carcinoma
}

\author{
Hong-Mei Gao', Wen-Bin Shen ${ }^{2}$, Xue-Yuan Zhang ${ }^{2}$, Jin-Rui Xu ${ }^{2}$, You-Mei Li ${ }^{2}$, Shu-Guang Li ${ }^{2}$, \\ Shu-Chai Zhu ${ }^{2}$ \\ ${ }^{1}$ Department of Radiation, Shijiazhuang People's Hospital, Shijiazhuang, China; ${ }^{2}$ Department of Radiation Oncology, the Fourth Hospital of Hebei \\ Medical University, Shijiazhuang, China \\ Contributions: (I) Conception and design: HM Gao; (II) Administrative support: WB Shen; (III) Provision of study materials or patients: JR Xu, YM \\ Li; (IV) Collection and assembly of data: HM Gao, SG Li; (V) Data analysis and interpretation: WB Shen, HM Gao, SC Zhu; (VI) Manuscript \\ writing: All authors; (VII) Final approval of manuscript: All authors. \\ Correspondence to: Wen-Bin Shen, MD. Department of Radiation Oncology, The Forth Hospital of Hebei Medical University, No. 12 Jiankan Road, \\ Chang'an District, Shijiazhuang 050011, China. Email: wbshen1979@sina.com.
}

Background: To explore the benefits of different types of irradiation on patients with postoperative locoregional recurrence (LRR) of thoracic esophageal squamous cell carcinoma (ESCC).

Methods: We analyzed the medical records of 344 patients with recurrent esophageal cancer (EC) at the Fourth Hospital of Hebei Medical University. All patients met an inclusion criteria that included having postoperative LRR (without distant metastasis), and having received either chemotherapy, radiotherapy, or chemoradiotherapy after LRR. Patients either received elective nodal irradiation (ENI) or involved field irradiation (IFI), with a stratified analysis performed on both groups. SPSS 19.0 software (IBM Corporation, Armonk, NY USA) was then used for statistical analysis.

Results: The median overall survival time of all patients after surgery was 33 months [95\% confidence interval (CI): 28.3-37.7 months]; the median overall survival time of patients after recurrence after radiotherapy was 12.8 months (95\% CI: $11.3-14.3$ months). There were 276 cases $(80.2 \%)$ of single local recurrence after surgery, and 68 cases $(19.8 \%)$ of multiple local recurrence $(\geq 2)$. The results of our multivariate analysis showed that the patient's gender, log odds of positive lymph nodes (LODDS), and the number of courses of chemotherapy were all independent factors affecting the patient's prognosis $(\mathrm{P}=0.003$, $\mathrm{P}<0.001$, and $\mathrm{P}<0.001)$. The results of stratified analysis showed that patients with esophageal lesion length $<5.0 \mathrm{~cm}$, stage N0, $\leq 9$ surgically dissected lymph nodes, no positive regional lymph node metastasis (LNM), and LODDS $\leq 0.030$ could benefit from ENI treatment $\left(X^{2}=4.208, P=0.032 ; X^{2}=6.262, P=0.012 ; X^{2}=10.359\right.$, $\left.\mathrm{P}=0.001 ; \mathrm{X}^{2}=6.327, \mathrm{P}=0.012 ; \mathrm{X}^{2}=6.026, \mathrm{P}=0.014\right)$; and patients with $\geq 16$ surgically dissected lymph nodes could benefit from IFI treatment $\left(\mathrm{X}^{2}=4.429, \mathrm{P}=0.035\right)$.

Conclusions: Chemotherapy, radiotherapy, and chemoradiotherapy are all effective modes of treatment for patients with postoperative LRR of EC. Patients with shorter esophageal lesions determined by preoperative esophagography, earlier postoperative pathological $\mathrm{N}$ staging, lower LODDS scores, and fewer surgically dissected lymph nodes might benefit more from ENI treatment than from IFI. However, patients with a larger number of lymph nodes dissected during surgery might benefit more from IFI treatment. To further confirm this study's conclusions, multiple prospective studies should be undertaken in the future.

Keywords: Esophageal neoplasms/esophageal squamous cell carcinoma (ESCC); surgical treatment; postoperative recurrence; types of irradiation; stratified analysis 
Submitted Jun 21, 2021. Accepted for publication Sep 03, 2021.

doi: 10.21037/apm-21-2080

View this article at: https://dx.doi.org/10.21037/apm-21-2080

\section{Introduction}

In patients with esophageal squamous cell carcinoma (ESCC), locoregional recurrence (LRR) is the main reason for treatment failure (1-4). For those with postoperative recurrent ESCC, surgical resection, radiotherapy, chemotherapy, or chemoradiotherapy are the key treatments in clinical practice. The suitability of each of these treatments varies from patient to patient, and each produces diverse therapeutic effects (5-8). Due to the range of recurrent regions and a patient's willingness to undergo a second operation, chemoradiotherapy is currently the most common treatment for ESCC patients. However, there has been no consensus on the radiotherapy target volume for postoperative recurrent ESCC. Discrepancies are present in the understanding of the postoperative target volume in various hospitals and medical institutions across the country. Theoretically, the volume of elective nodal irradiation (ENI) is greater than that of involved field irradiation (IFI), which may increase the control rate of regional lymph nodes, as well as the incidence of toxicities (9-12). In our cancer center at the Fourth Hospital of Hebei Medical University, radiotherapy with or without chemotherapy remains a key treatment for patients with postoperative recurrentt esophageal cancer (EC). To further clarify the advantages and disadvantages of the two different irradiation methods, we performed a retrospective analysis on a diverse group of 344 patients experiencing postoperative recurrent EC after chemoradiotherapy. We present the following article in accordance with the STROBE reporting checklist (available at https://dx.doi.org/10.21037/apm-21-2080).

\section{Methods}

Each patient included in the study met the following inclusion criteria: (I) patient underwent radical surgery for EC (with no adjuvant chemoradiotherapy before or after surgery) in the Department of Thoracic Surgery, Fourth Hospital of Hebei Medical University; (II) thoracic ESCC was confirmed by postoperative pathology; (III) pT1-4N0$3 \mathrm{M} 0 \mathrm{EC}$ was diagnosed according to the eighth edition of the tumor-node-metastasis (TNM) staging system outlined by the American Joint Committee on Cancer (AJCC)/ the Union for International Cancer Control (UICC); (IV) first recurrence occurred at the time of enrollment; (V) the Karnofsky score was $\geq 70$ points; (VI) multiple and simultaneous LRR in the thoracic cavity was detected, but distant metastasis was not; (VII) patient showed no serious underlying diseases affecting treatment and had undergone chemoradiotherapy with a radiation dose of $\geq 45 \mathrm{~Gy}$; and (VIII) no other malignant tumors were present. The study was conducted in accordance with the Declaration of Helsinki (as revised in 2013). The study was approved by the Ethics Committee of the Fourth Hospital of Hebei Medical University (2021KT254) and individual consent for this retrospective analysis was waived.

General clinicopathological data were collected from patients with EC who were treated at the Fourth Hospital of Hebei Medical University from January 2009 to December 2014. Among them, 344 cases (male-to-female ratio, 2.5:1) were eligible for enrollment. The median age at surgery was 59 years (age range, 39-79 years), and the median age of patients with postoperative recurrent EC receiving chemoradiotherapy was 60.2 years (age range, $39.8-81.9$ years). There were 83,15 , and 15 patients with a past history of hypertension, diabetes, and coronary heart disease, respectively. The length of lesion determined by esophageal barium meal examination before surgery was $1.0-11.0 \mathrm{~cm}$ (median $5.0 \mathrm{~cm}$ ). The main surgical methods included a left thoracotomy for 292 cases (84.9\%), a right thoracotomy for 26 cases $(7.6 \%), 2$ incisions (neck and abdomen) for 13 cases (3.8\%), and 3 incisions (neck, chest, and abdomen) for 13 cases (3.8\%). In addition, postoperative pathology showed positive stumps in 30 cases, and postoperative pathology showed no nerve invasion. This general information is listed in Table 1.

For our study, postoperative LRR was detected by anastomotic recurrence (AR) and lymph nodal recurrence. AR was confirmed by a biting biopsy analyzed under electron gastroscopy. Superficial lymph node metastasis (LNM) was confirmed pathologically by a needle biopsy, and the diagnosis of LNM in the remaining regions was confirmed by computed tomography (CT), magnetic resonance imaging (MRI), positron emission tomography/ computed tomography (PET/CT), or B-ultrasound.

The location of recurrent lymph nodes was mainly 
Table 1 Results of univariate analysis of prognostic factors in 344 postoperative LRR EC patients

\begin{tabular}{|c|c|c|c|c|c|c|c|}
\hline Factor & $\mathrm{N}$ & \multicolumn{3}{|c|}{ Survival rate (\%) } & MST (M) & $x^{2}$ & $P$ \\
\hline Sex & & & & & & 10.814 & 0.001 \\
\hline Male & 247 & 47.2 & 18.2 & 12.2 & 11.3 & & \\
\hline Female & 97 & 70.1 & 33.5 & 26.6 & 18.9 & & \\
\hline$\geq 60$ & 163 & 53.2 & 22.3 & 11.6 & 12.7 & & \\
\hline Underlying diseases & & & & & & 0.293 & 0.588 \\
\hline No & 247 & 52 & 22.3 & 18.4 & 12.8 & & \\
\hline Yes & 178 & 45.9 & 17 & 13.3 & 11.1 & & \\
\hline History of drinking & & & & & & 8.468 & 0.004 \\
\hline No & 200 & 59 & 28.6 & 20.1 & 15 & & \\
\hline Yes & 144 & 45.7 & 13.6 & 10.9 & 11 & & \\
\hline Family history & & & & & & 2.412 & 0.120 \\
\hline No & 293 & 53.3 & 25.4 & 18.3 & 13.4 & & \\
\hline Yes & 51 & 54.5 & 3.8 & 3.8 & 12.4 & & \\
\hline Differentiation & & & & & & 0.434 & 0.510 \\
\hline Lower & 117 & 54.6 & 11.3 & 9.4 & 12.6 & & \\
\hline Types of thoracotomy & & & & & & 0.714 & 0.398 \\
\hline Left thoracotomy & 292 & 53.1 & 23.6 & 16.9 & 12.7 & & \\
\hline Other types & 52 & 56.1 & 16.3 & 13.1 & 13.9 & & \\
\hline \multicolumn{3}{|c|}{ Length of esophageal lesion determined by esophagography (cm) } & & & & 9.287 & 0.002 \\
\hline$<5.0$ & 150 & 59.2 & 29.5 & 25 & 18.9 & & \\
\hline$\geq 5.0$ & 194 & 49.1 & 17.6 & 10.8 & 11.8 & & \\
\hline pT staging & & & & & & 8.257 & 0.041 \\
\hline pT1 & 45 & 55.6 & 28.1 & 24.6 & 17.8 & & \\
\hline pT2 & 69 & 68.5 & 32.2 & 25.2 & 19.4 & & \\
\hline pT3 & 220 & 48.7 & 18.7 & 13.4 & 11.8 & & \\
\hline pT4 & 10 & 40 & 10 & 10 & 5.9 & & \\
\hline
\end{tabular}

Table 1 (continued) 
Table 1 (continued)

\begin{tabular}{|c|c|c|c|c|c|c|c|}
\hline Factor & $\mathrm{N}$ & \multicolumn{3}{|c|}{ Survival rate (\%) } & MST (M) & $x^{2}$ & $\mathrm{P}$ \\
\hline pN staging & & & & & & 8.256 & 0.016 \\
\hline $\mathrm{pNO}$ & 175 & 57.5 & 30.5 & 22.6 & 13.9 & & \\
\hline $\mathrm{pN} 1$ & 113 & 49.4 & 14.6 & 10.7 & 11.8 & & \\
\hline Negative & 314 & 53.7 & 22.9 & 16.6 & 12.7 & & \\
\hline Positive & 30 & 52.3 & 20.7 & 20.7 & 13.8 & & \\
\hline Vessel carcinoma embolus & & & & & & 3.524 & 0.06 \\
\hline No & 325 & 54.3 & 23.9 & 17.1 & 13.4 & & \\
\hline$\leq 9$ & 139 & 52.4 & 24.3 & 17.8 & 12.6 & & \\
\hline $10-15$ & 126 & 54 & 20.1 & 14.7 & 12.7 & & \\
\hline$\geq 16$ & 79 & 54.7 & 22.9 & 16.6 & 12.8 & & \\
\hline LODDS & & & & & & 21.63 & 0 \\
\hline$\leq 0.030$ & 194 & 61.2 & 32.3 & 23.9 & 14.7 & & \\
\hline$>0.030$ & 150 & 43.6 & 11.2 & 7.5 & 9.8 & & \\
\hline Postoperative chemotherapy & & & & & & 0.012 & 0.912 \\
\hline No & 156 & 50.2 & 25.8 & 16.2 & 12.2 & & \\
\hline IFI & 214 & 53.4 & 21.5 & 13.5 & 12.5 & & \\
\hline ENI & 130 & 53.9 & 24.3 & 20.8 & 13.9 & & \\
\hline Irradiation dose (Gy) & & & & & & 10.446 & 0.005 \\
\hline$<60$ & 47 & 40 & 10.3 & 6.9 & 9 & & \\
\hline 60 & 186 & - & 27.7 & 22.9 & 14 & & \\
\hline$>60$ & 111 & 52.2 & 19.1 & 11.4 & 12.3 & 111 & \\
\hline Interval between surgery and recurrence (month) & & & & & & 22.605 & 0 \\
\hline$\leq 24$ & 262 & 48.2 & & 16 & 12 & 11.6 & \\
\hline$>24$ & 82 & 70.1 & & 42.5 & 26.6 & 28.9 & \\
\hline No. of recurrent regions after surgery & & & & & & 0.784 & 0.376 \\
\hline 1 & 276 & 55.6 & & 30.6 & 17 & 13.4 & \\
\hline$\geq 2$ & 68 & 44.7 & & 18.6 & 13.9 & 10.9 & \\
\hline
\end{tabular}

LRR, locoregional recurrence; EC, esophageal cancer; MST, median survival time; LODDS, log odds of positive lymph nodes; IFI, involved field irradiation; ENI, elective nodal irradiation. 
defined according to the eighth edition TNM staging system for EC. This included stations $1-8 \mathrm{M}$ being defined as lymph nodes in the middle and upper mediastinal region (the upper boundary included the supraclavicular region, tracheoesophageal groove, and the paratracheal region at the level of the lung apex to the lower edge of the inferior pulmonary vein); stations $8 \mathrm{Lo}, 9$, and 15 being defined as lymph nodes in the inferior mediastinal region (the upper boundary was the lower edge of the lymph node drainage area in the middle and upper mediastinal region, and the lower boundary was at the top of the diaphragm); and stations 16-20 being defined as the upper abdominal lymph node area (the upper boundary was connected to the lower boundary of the lymphatic drainage area in the inferior mediastinal region, and the lower boundary was at the level of the abdominal trunk).

All patients underwent intensity-modulated radiation therapy (IMRT) with $6 \mathrm{MV}$ X-rays. For the 214 patients who received IFI, the target volume included recurrent lymph nodes or recurrent tumor tissues and was expanded by $2-3 \mathrm{~cm}$ margins (avoiding damage to surrounding normal tissues and vital organs). The prescribed dose ranged from 45-70 Gy (median $60 \mathrm{~Gy}$ ). The remaining 130 patients underwent ENI using the same target volume as IFI. In addition, preventive irradiation was performed on the corresponding lymph node drainage areas of patients with recurrence in different segments. For patients with recurrence in the upper thoracic segment, drainage areas encompassed the bilateral supraclavicular region and paraesophageal region (zones 2, 4, 5 , and 7). As for those with recurrence in the middle thoracic segment, drainage areas included the paraesophageal region (zones 2, 4, 5, and 7). For recurrence in the lower thoracic segment, drainage areas encompassed the paraesophageal region (zones 2, 4, 5, 7, and 8), paracardial area, and left gastric area. The upper and lower boundaries of the target volume were adjusted according to the anatomical barrier and the recurrent regions. In addition, 74 patients were initially treated 18-28 times with conventional segmented irradiation (36-50.4 Gy) in the drainage area, and the target volume was localized for late course accelerated hyperfractionated irradiation, with a dose of 12-24 Gy (612 times). For all patients with lymph nodal recurrence in the supraclavicular region, if there was any residual after IMRT, the residual part was irradiated with an electronic beam. All patients in this group completed the treatment according to the clinical treatment regimen.

A total of 188 patients $(54.7 \%)$ also received a regimen of chemotherapy, which was mainly platinum-based combination chemotherapy. The number of courses of this treatment varied from 1-7 (median 3), with 112 patients receiving $\geq 3$ courses. All 188 patients received concurrent chemotherapy, with several patients also undergoing radiotherapy prior to this.

Patient follow-up was mainly performed by combining a telephone interview or outpatient visit with case data. The follow-up ranged from the day the patient received either chemotherapy, radiotherapy, or chemoradiotherapy for postoperative LRR up to December 31, 2019. The patients were re-examined every 3-6 months in the first year, and every 6-12 months thereafter. Thirteen cases did not take part in the follow-ups (3.8\%) and were subsequently censored at the last date of follow-up.

\section{Statistical analysis}

Statistical analysis was undertaken using SPSS 19.0 software (IBM Corporation, Armonk, NY, USA). The comparison of numerical data was performed using a chisquare test. A log-rank test and the Kaplan-Meier (KM) method were used for the univariate survival analysis, while a Cox proportional hazard model was used for multivariate analysis. Propensity score matching (PSM) was used to test patient results under different types of irradiation. Patients were divided into groups to reduce confounding bias. The level of significance was set at $\alpha=0.05$, and a $\mathrm{P}$ value $<0.05$ was considered statistically significant.

\section{Results}

To ensure the success of the number of lymph nodes dissected during the operation, we used the log odds of positive lymph nodes (LODDS) system. The calculation method of LODDS was as follows (13): $\log$ (the number of positive lymph nodes +0.5$) /($ the number of negative lymph nodes + 0.5). The subsequent LODDS value in this study ranged from -1.204 to 0.638 (median 0.030 ).

The 1-, 3-, and 5-year overall survival (OS) rates of all patients after postoperative radiotherapy (PORT) were $91.3 \%, 47.1 \%$, and $32.2 \%$, respectively [median 33 months; 95\% confidence interval (CI): 28.3-37.7 months]. The 1-, 3 -, and 5-year OS rates of patients with LRR after PORT were $53.6 \%, 22.6 \%$, and $16.4 \%$, respectively (median 12.8 months; 95\% CI: 11.3-14.3 months). The 1-, 3-, and 5 -year recurrence-free survival (RFS) rates after PORT were $46.5 \%, 16.9 \%$, and $12.0 \%$, respectively (median 11.0 months; 95\% CI: 9.6-12.4 months). The 1-, 3-, and 
Table 2 Multivariate analysis results of prognostic factors in 344 postoperative LRR EC patients

\begin{tabular}{|c|c|c|c|c|c|c|c|}
\hline Factor & B & SE & Wald & $\mathrm{P}$ & $\mathrm{HR}$ & \multicolumn{2}{|c|}{$95.0 \% \mathrm{Cl}$} \\
\hline Sex & -0.448 & 0.151 & 8.839 & 0.003 & 0.639 & 0.475 & 0.858 \\
\hline LODDS & 0.374 & 0.084 & 19.630 & 0.000 & 1.454 & 1.232 & 1.715 \\
\hline No. of courses of chemotherapy & -0.531 & 0.144 & 13.666 & 0.000 & 0.588 & 0.444 & 0.779 \\
\hline
\end{tabular}

LRR, locoregional recurrence; EC, esophageal cancer; Cl, confidence interval; LODDS, log odds of positive lymph nodes.

5 -year progression-free survival (PFS) rates after PORT were $39.8 \%, 11.3 \%$, and $6.7 \%$, respectively (median 7.9 months; $95 \%$ CI: $5.8-10.0$ ).

The analysis results of all patients with postoperative LRR found the interval between surgery and recurrence was $0.3-87.4$ months (median 11.6 months). Based on the distribution of specific recurrent regions, the results were as follows: 50 (14.5\%) cases had simple supraclavicular lymph node recurrence (SCLNR), 182 (52.9\%) cases had simple mediastinal lymph node (MLN) recurrence, 23 (6.7\%) cases had simple AR; 21 (6.1\%) cases had simple abdominal lymph node recurrence (ALNR); 21 (6.1\%) cases had both SCLNR and MLN; 12 (3.5\%) cases had both SCLNR and ALNR; 4 (1.2\%) cases had both SCLNR and AR; 16 (4.7\%) cases had both MLN and AR; 9 (2.6\%) cases had both MLN and ALNR; 1 (0.3\%) case had both ALNR and AR; 3 (0.9\%) cases had SCLNR, MLN, and ALNR; and 2 (0.6\%) cases had SCLNR, MLN, and AR.

The results of our univariate analysis of all patients showed that the patient's gender, smoking history, drinking history, esophageal lesion length determined by angiography, pathological $\mathrm{T}$ staging, pathological $\mathrm{N}$ staging, LODDS, number of courses of chemotherapy, irradiation dose, interval between surgery, and recurrence were risk factors affecting patient prognosis $\left(X^{2}=10.814\right.$, $\mathrm{P}=0.001 ; \mathrm{X}^{2}=6.091, \mathrm{P}=0.014 ; \mathrm{X}^{2}=8.468, \mathrm{P}=0.004 ; \mathrm{X}^{2}=9.286$, $\mathrm{P}=0.002 ; \mathrm{X}^{2}=8.257, \mathrm{P}=0.041 ; \mathrm{X}^{2}=8.256, \mathrm{P}=0.016 ; \mathrm{X}^{2}=21.630$, $\mathrm{P}<0.001 ; \mathrm{X}^{2}=13.957, \mathrm{P}<0.001 ; \mathrm{X}^{2}=10.446, \mathrm{P}=0.005$; $\left.\mathrm{X}^{2}=22.605 \mathrm{P}<0.001\right)$. Detailed information of this analysis is listed in Table 1.

We conducted a multivariate analysis of all patients by entering all statistically significant factors identified by the univariate analysis into a Cox multivariate analysis model. The results revealed that the patient's gender, LODDS, and the number of courses of chemotherapy were all independent factors affecting the prognosis of the patient $(\mathrm{P}=0.003, \mathrm{P}<0.001, \mathrm{P}<0.001)$. Detailed information of this analysis is listed in Table 2.
Our stratified analysis compared the two different types (ENI and IFI) of irradiation used in our study, and the general clinical and pathological indicators of the two groups were compared. The results suggested that there was a statistically significant difference in the composition ratio between the method of thoracotomy $\left(X^{2}=22.703\right.$, $\mathrm{P}=0.000)$ and the length of lesion determined by esophageal barium meal examination in both groups $\left(X^{2}=9.527\right.$, $\mathrm{P}=0.002$ ). According to the results of Cox multivariate analysis, the PSM method was used to achieve a balanced distribution of patients in the two groups (1:1). The results showed that there were 130 patients in each group, and that the composition ratio of the two groups was balanced, indicating no significant difference. Detailed information of this analysis is listed in Table 3.

The subsequent results showed that patients who had any of the following could benefit from ENI treatment: an esophageal lesion length $<5.0 \mathrm{~cm}, \leq 9$ surgically dissected lymph nodes, 0 positive regional LNM, $\leq 0.030$ LODDS, or N0 stage $\left(\mathrm{X}^{2}=4.208, \mathrm{P}=0.032 ; \mathrm{X}^{2}=6.262, \mathrm{P}=0.012\right.$; $\mathrm{X}^{2}=10.359, \mathrm{P}=0.001 ; \mathrm{X}^{2}=6.327, \mathrm{P}=0.012 ; \mathrm{X}^{2}=6.026$, $\mathrm{P}=0.014)$. We also found that patients with $\geq 16$ surgically dissected lymph nodes could benefit from IFI treatment $\left(\mathrm{X}^{2}=4.429, \mathrm{P}=0.035\right)$. Detailed information of this analysis is listed in Table 4.

\section{Discussion}

LRR is the main cause for treatment failure in ESCC patients. Chemotherapy, radiotherapy, and chemoradiotherapy are effective and safe treatment options widely used in clinical practice $(14,15)$. However, in clinical application, there has been no consensus regarding whether ENI or IFI should be applied to these patients. Currently, there are only a few reports which stipulate the volume of the target area for patients receiving irradiation for postoperative LRR of EC. Unfortunately, these studies exhibit several disadvantages, including analyzing only a small number of EC patients, not 
Table 3 The composition ratio of patients under different irradiation types before and after PSM

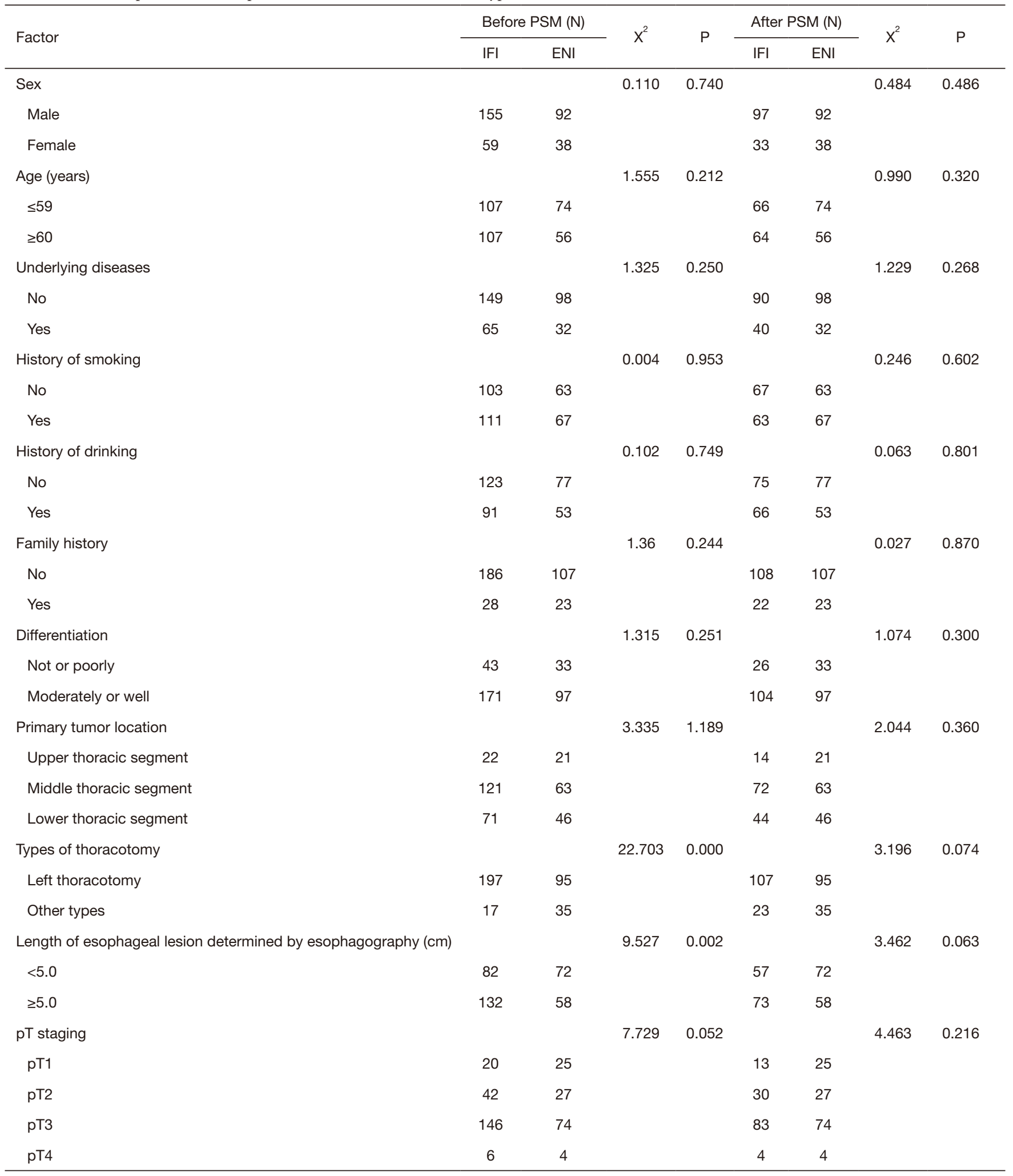

Table 3 (continued) 
Table 3 (continued)

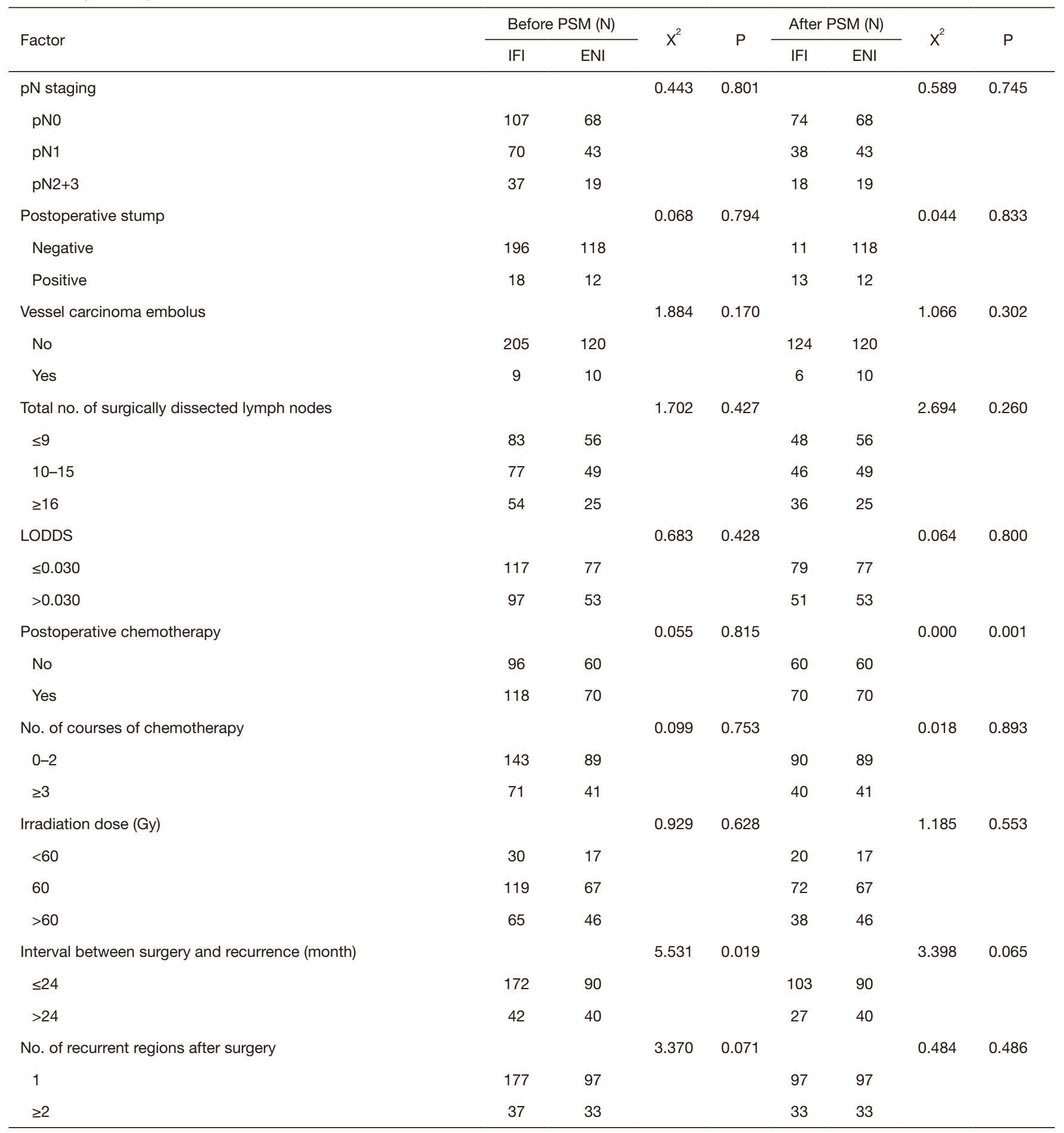

PSM, propensity score matching; IFI, involved field irradiation; ENI, elective nodal irradiation; LODDS, log odds of positive lymph nodes. 
Table 4 Analysis results of the impact of different irradiation types on patient prognosis

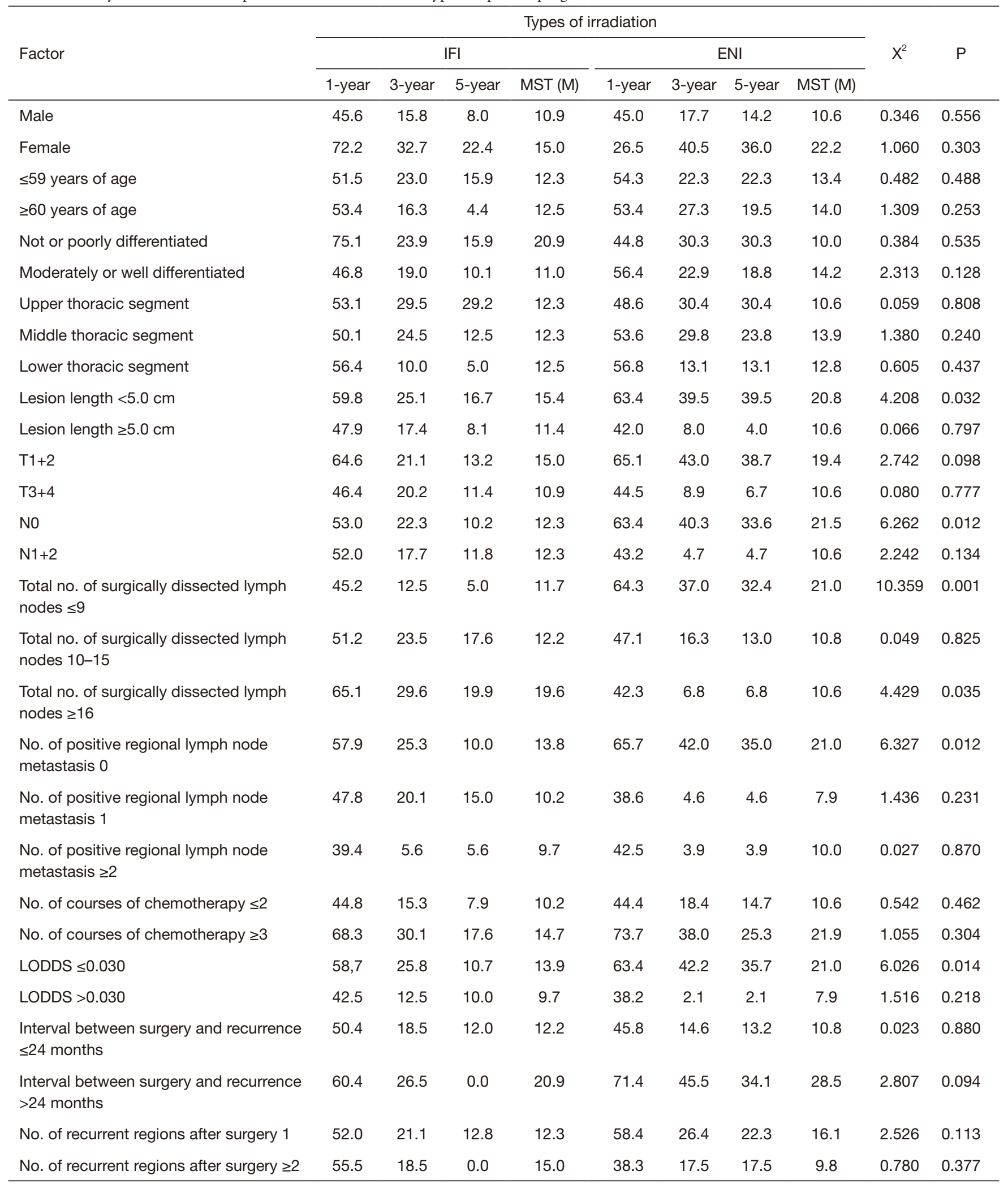

IFI, involved field irradiation; ENI, elective nodal irradiation; MST, median survival time; LODDS, log odds of positive lymph nodes. 
undertaking a stratified analysis of most patients, and (as in most studies) failing to comprehensively compare the efficacy of different irradiation methods on patient prognosis. For our study, we conducted a retrospective analysis of 344 patients with postoperative LRR of EC at a single center. The results of our univariate and multivariate analyses showed that the mode of radiotherapy (ENI or IFI) was not a prognostic factor in this group of patients. To clarify prognosis of different patients under different types of irradiation, we conducted a stratified analysis. The results indicated that ENI treatment was beneficial to patients with early lesions, specifically patients with an esophageal lesion length less than $5.0 \mathrm{~cm}$, pathological stage $\mathrm{T} 1+2$, stage N0, and LODDS $\leq 0.030$. In addition, patients with $\leq 9$ surgically dissected lymph nodes could also benefit from ENI, whereas patients with $\geq 16$ surgically dissected lymph nodes and LODDS $>0.030$ could benefit from IFI treatment. Taken together, the choice of irradiation mode for treating patients with postoperative LRR of EC can vary from person to person, and different groups of people might benefit more from ENI, IFI, or other radiotherapy treatments.

Currently, there is no consensus on the delineation of the target area for patients receiving salvage radiotherapy for LRR of EC. Unlike older forms of two-dimensional radiotherapy, the wide clinical application of precision radiotherapy technology requires the precise delineation of the target area in clinical practice. However, to our knowledge, there are few related reports on this subject, and these report diverse conclusions. Jingu et al. (12) analyzed 30 patients with postoperative LRR EC; a T-shaped field (including bilateral supraclavicular, mediastinal and abdominal regions) was used for 11 patients, and IFI was used for the remaining 19 patients. The results showed that other than benefiting patients, irradiation with a T-shaped field was more likely to lead to acute adverse events of grade 3 or higher. In another of their related studies (16), they analyzed 35 patients with postoperative recurrent EC, with a T-shaped field used for 15 patients and IFI used for the remaining 20. Among them, 2 patients who underwent irradiation with a T-shaped field experience other types of LNM. In another study (14), they analyzed 80 patients with postoperative LRR of EC who received chemoradiotherapy. In this study, the interval between surgery, recurrence, and $\mathrm{AR}$ were independent factors affecting the prognosis of patients. After a matched-pair analysis, they found 26 patients in both the IFI and ENI groups. The OS rate and local control (LC) rate of the patients in the IFI and ENI groups were $50.9 \%$ vs. $21.1 \%(\mathrm{P}=0.016)$ and $79.0 \%$ vs.
42.2\% ( $\mathrm{P}=0.014)$, respectively. ENI was therefore considered unnecessary for patients with postoperative LRR of EC. In a study conducted by Kawamoto et al. (5), the prognosis of 21 patients with lymph node recurrence (LNR) after surgery for EC were retrospectively evaluated. The 2-year OS rate of these patients was $78 \%$. After treatment, $9(42.9 \%)$ patients were found to have failed, including 4 patients (19\%) with recurrence within the irradiation field, while another $5(23.8 \%)$ patients had distant metastases. The authors indicated that IFI was an effective radiotherapy regimen for patients with postoperative LRR of EC. In another study by Kawamoto et al. (8), they retrospectively analyzed 57 patients with postoperative LRR of EC who received radiotherapy, including $15(26.3 \%)$ patients who received ENI, and 42 (73.7\%) patients who received IFI; the median survival period of the two groups was 21 and 22 months, respectively $(\mathrm{P}=0.38)$. In the abovementioned studies, the researchers asserted that preventive irradiation should not be recommended for patients with LRR of EC. However, these studies have several disadvantages, including the small number of patients who took part in their studies, the amount of patients with solitary LNR, a T-shaped field design that was different from the current delineation standard of an elective nodal drainage area under precise treatment, and a lack of a stratified analysis across a diverse group of patients. To add to this research, our study results show that different types of irradiation have no significant effect on the number of recurrent regional lymph nodes. Therefore, we believe that for different types of patients, a further stratified analysis should be carried out to pinpoint which patients will benefit more from a given irradiation field.

Additional research, such as that by Zhang et al. (11) also retrospectively analyzed the clinical data of 50 patients. This group (except for SCLNR patients who received irradiation of the supraclavicular lymphatic drainage area) underwent IFI, with 1-year and 3-year survival rates of $56 \%$ and $14 \%$, respectively. The median PFS and OS rate were 9.8 and 13.3 months, respectively. Unfortunately, this study failed to further compare and analyze different types of irradiation. Chen et al. (15) also retrospectively analyzed the survival rate and prognostic factors of 83 patients with local LNR after radical resection of ESCC. Among them, 41 cases received radiotherapy alone, 42 cases received radiotherapy combined with chemotherapy, and all received IMRT. In addition to the target volume of the target area, the adjacent drainage area was irradiated. The 1-, 3-, and 5-year OS rates of all patients were $83.0 \%, 40.1 \%$, and $35.1 \%$, respectively, with a median survival period of 
18 months (range, 5-75 months). However, these authors did not compare the efficacy of different irradiation fields in patients. In our study, we retrospectively analyzed 344 patients with postoperative LRR of EC, including 130 patients who received ENI, and 214 patients who received IFI. The 1-, 3-, and 5-year OS rates of patients receiving ENI and IFI were $53.9 \%, 24.3 \%, 20.8 \%$, and $53.4 \%, 21.5 \%, 13.5 \%$, respectively, with a median survival period of 13.9 months for ENI patients and 12.5 months for IFI patients. Although no significant difference was found between the two groups, the 5-year survival rate of the two groups indicated that patients receiving ENI might have a more advantageous long-term prognosis.

The length of esophageal lesion and postoperative pathological staging are important factors affecting the prognosis of patients with EC after surgery (17). Most research results show that the lesion length of EC is closely related to the postoperative pathological stage $\mathrm{T}$ and $\mathrm{N}$, and that patients with shorter lesions have relatively early $\mathrm{T}$ and $\mathrm{N}$ staging $(18,19)$. According to the calculation formula of LODDS, it is clear that LODDS is related to the number of lymph nodes, with smaller LODDS correlating to the earlier stages of a patient's disease. The location, size, mobility, relationship with surrounding organs, and local LNM of esophageal tumors are closely related to the scope of surgery. Therefore, the patient's preoperative condition directly affects the extent of resection and lymph node dissection during surgery. In the case of resection, the extent of resection is often relatively large for patients with an advanced illness. Compared with a smaller area, a larger surgical area will inflict considerable trauma to the patient and lead to a variety of unpredictable surgical complications, as well as a longer period of recovery and recuperation (20).

Immune function status is known to be an important indicator that affects the prognosis of patients with malignant tumors. Numerous researchers $(21,22)$ believe that the occurrence and development of tumors are caused by low immunity or weakened immunogenicity of mutant cells. Changes in the number and function of immunocytes may lead to immune function disorder and resultant tumors. Andaluz-Ojeda et al. (23) even believe that tumor staging, patient survival time, and the prognostic effect of surgery could be estimated by a T lymphocyte count, T lymphocyte subgroup count, and the degree of the disorder. Surgery has a dual effect on the immune function of patients. On the one hand, surgical trauma, anesthesia, and blood transfusion can lead to decreased immunity to a certain extent, but on the other hand, with the recovery of surgical trauma, the immune function of patients with $\mathrm{EC}$ can be improved (24). Moreover, postoperative chemoradiotherapy for EC may result in T cell immunosuppression and the compromised function of immune cells, thereby resulting in a prolonged period of lower immune function of the $\mathrm{T}$ cells and adversely affecting the recovery of a patient's immune functions. This suggests that different treatment methods have selective effects on the recovery of immune function (25). For example, EC patients in more advanced stages often have lower local and systemic immune function. For patients whose immune function has not fully recovered, larger irradiation fields may contribute to a further decline in the patient's immunity and affect the prognosis, thereby offsetting the benefits of large irradiation fields. In addition, patients with late-stage EC and those with lower immunity are more likely to have distant metastasis. This means that even larger irradiation fields can only serve as a local treatment option and cannot reduce the rate of distant metastasis. This may explain why the stratified analysis results of this study show that after ENI treatment, prognosis is more favorable in patients at the early-stage of EC than in patients at the advanced-stage.

This study has several limitations. First, as this study is retrospective, there is an inherent bias in the cases selected. Second, in addition to the effects of different irradiation fields, there are many factors effecting the treatment and prognosis of patients with postoperative LRR of EC, including radiation dose, chemotherapy regimen, and the timing of chemotherapy treatment. Third, due to word count limitations, this study did not conduct further analysis on the toxic effects, other side effects, or the recurrence patterns of the two types of irradiation. Lastly, due to the limited amount of literature available on using irradiation fields for treating patients with postoperative LRR of EC, discussion and comparison with other relevant findings were limited in this paper.

In conclusion, chemotherapy, radiotherapy, or chemoradiotherapy are effective treatment modes for patients with postoperative LRR of EC in the thoracic cavity, but different types of irradiation fields may benefit different patients in clinical settings. In respect to survival, patients who would benefit the most from ENI therapy were found to have a shorter esophageal lesion (determined through preoperative esophagography), early postoperative $\mathrm{T} / \mathrm{N}$ staging, lower LODDS score, and a small number of lymph nodes dissected, whereas patients with a larger number of lymph nodes dissected during surgery and a higher LODDS score were found to benefit more from 
IFI. To further confirm these results, a large number of prospective controlled studies should be conducted in the future.

\section{Acknowledgments}

Funding: None.

\section{Footnote}

Reporting Checklist: The authors have completed the STROBE reporting checklist. Available at https://dx.doi. org/10.21037/apm-21-2080

Data Sharing Statement: Available at https://dx.doi. org/10.21037/apm-21-2080

Conflicts of Interest: All authors have completed the ICMJE uniform disclosure form (available at https://dx.doi. org/10.21037/apm-21-2080). The authors have no conflicts of interest to declare.

Ethical Statement: The authors are accountable for all aspects of the work in ensuring that questions related to the accuracy or integrity of any part of the work are appropriately investigated and resolved. The study was conducted in accordance with the Declaration of Helsinki (as revised in 2013). The study was approved by the Ethics Committee of the Fourth Hospital of Hebei Medical University (2021KT254) and individual consent for this retrospective analysis was waived.

Open Access Statement: This is an Open Access article distributed in accordance with the Creative Commons Attribution-NonCommercial-NoDerivs 4.0 International License (CC BY-NC-ND 4.0), which permits the noncommercial replication and distribution of the article with the strict proviso that no changes or edits are made and the original work is properly cited (including links to both the formal publication through the relevant DOI and the license). See: https://creativecommons.org/licenses/by-nc-nd/4.0/.

\section{References}

1. Hsu PK, Chen HS, Huang CS, et al. Patterns of recurrence after oesophagectomy and postoperative chemoradiotherapy versus surgery alone for oesophageal squamous cell carcinoma. Br J Surg 2017;104:90-7.
2. Yamashita K, Watanabe M, Mine S, et al. Patterns and Outcomes of Recurrent Esophageal Cancer After Curative Esophagectomy. World J Surg 2017;41:2337-44.

3. Lin G, Liu H, Li J. Pattern of recurrence and prognostic factors in patients with $\mathrm{pT} 1-3 \mathrm{~N} 0$ esophageal squamous cell carcinoma after surgery: analysis of a single center experience. J Cardiothorac Surg 2019;14:58.

4. Zhang WY, Chen XX, Chen WH, et al. Nomograms for predicting risk of locoregional recurrence and distant metastases for esophageal cancer patients after radical esophagectomy. BMC Cancer 2018;18:879.

5. Kawamoto T, Nihei K, Sasai K, et al. Involved-field chemoradiotherapy for postoperative solitary lymph node recurrence of esophageal cancer. Esophagus 2018;15:256-62.

6. Deng J, Xia Y, Chen Y, et al. Long term results of different radiotherapy techniques and fractions for esophageal squamous cell carcinoma. Transl Cancer Res 2020;9:2287-94.

7. Zhou YQ, Ding NX, Wang LJ, et al. Salvage radiochemotherapy for lymph node recurrence after radical surgery of esophageal cancer. Medicine (Baltimore) 2018;97:e9777.

8. Shang QX, Yang YS, Yuan Y, et al. Clinical and prognostic effects of adjuvant therapy on less advanced esophageal squamous cell carcinoma patients. Ann Palliat Med 2020;9:681-99.

9. Du D, Song T, Liang X, et al. Concurrent chemoradiotherapy with elective lymph node irradiation for esophageal cancer: a systemic review and pooled analysis of the literature. Dis Esophagus 2017;30:1-9.

10. Bao Y, Liu S, Zhou Q, et al. Three-dimensional conformal radiotherapy with concurrent chemotherapy for postoperative recurrence of esophageal squamous cell carcinoma: clinical efficacy and failure pattern. Radiat Oncol 2013;8:241.

11. Zhang J, Peng F, Li N, et al. Salvage concurrent radiochemotherapy for post-operative local recurrence of squamous-cell esophageal cancer. Radiat Oncol 2012;7:93.

12. Jingu K, Nemoto K, Matsushita $\mathrm{H}$, et al. Results of radiation therapy combined with nedaplatin (cisdiammine-glycoplatinum) and 5-fluorouracil for postoperative locoregional recurrent esophageal cancer. BMC Cancer 2006;6:50.

13. Wen J, Ye F, He X, et al. Development and validation of a prognostic nomogram based on the log odds of positive lymph nodes (LODDS) for breast cancer. Oncotarget 2016;7:21046-53. 
14. Jingu K, Umezawa R, Yamamoto T, et al. Elective nodal irradiation is not necessary in chemoradiotherapy for postoperative loco-regional recurrent esophageal cancer. Jpn J Clin Oncol 2017;47:200-5.

15. Chen J, Yin W, Yao H, et al. Salvage treatment for lymph node recurrence after radical resection of esophageal squamous cell carcinoma. Radiat Oncol 2019;14:169.

16. Jingu K, Ariga H, Nemoto K, et al. Long-term results of radiochemotherapy for solitary lymph node metastasis after curative resection of esophageal cancer. Int J Radiat Oncol Biol Phys 2012;83:172-7.

17. Yang Y, Jia J, Sun Z, et al. Prognosis impact of clinical characteristics in patients with inoperable esophageal squamous cell carcinoma. PLoS One 2017;12:e0182660.

18. Gong L, Yue J, Duan X, et al. Comparison of the therapeutic effects of endoscopic submucosal dissection and minimally invasive esophagectomy for T1 stage esophageal carcinoma. Thorac Cancer 2019;10:2161-7.

19. Vadhwana B, Zosimas D, Lykoudis PM, et al. Tumour length as an independent prognostic factor in resectable oesophageal carcinoma. Ann R Coll Surg Engl 2020;102:185-90.

20. Nakano T, Kamei T, Onodera Y, et al. Thoracoscopic surgery in the prone position for esophageal cancer in patients with situs inversus totalis: A report of two cases.

Cite this article as: Gao HM, Shen WB, Zhang XY, Xu JR, Li YM, Li SG, Zhu SC. Preliminary analysis of the benefits of different irradiation types on patients with postoperative locoregional recurrence of esophageal cell squamous carcinoma. Ann Palliat Med 2021;10(9):9467-9479. doi: 10.21037/apm-212080
Int J Surg Case Rep 2017;31:43-6.

21. Nishijima TF, Muss HB, Shachar SS, et al. Prognostic value of lymphocyte-to-monocyte ratio in patients with solid tumors: A systematic review and meta-analysis. Cancer Treat Rev 2015;41:971-8.

22. Gu L, Li H, Chen L, et al. Prognostic role of lymphocyte to monocyte ratio for patients with cancer: evidence from a systematic review and meta-analysis. Oncotarget 2016;7:31926-42.

23. Andaluz-Ojeda D, Iglesias V, Bobillo F, et al. Early levels in blood of immunoglobulin $M$ and natural killer cells predict outcome in nonseptic critically ill patients. J Crit Care 2013;28:1110.e7-1110.e10.

24. Ding H, Xu J, You J, et al. Effects of enteral nutrition support combined with enhanced recovery after surgery on the nutritional status, immune function, and prognosis of patients with esophageal cancer after Ivor-Lewis operation. J Thorac Dis 2020;12:7337-45.

25. Baba Y, Nomoto D, Okadome K, et al. Tumor immune microenvironment and immune checkpoint inhibitors in esophageal squamous cell carcinoma. Cancer Sci 2020;111:3132-41.

(English Language Editors: J. Goetz and J. Gray) 